\title{
An Analytical Analysis of Path Loss Models FOR MOBILE CELLULAR WIRELESS COMMUNICATIONS
}

\author{
Md. Shariful Islam ${ }^{*}$, Md. Ashek Raihan Mahmud ${ }^{1}$, \\ Md. Jashim Uddin ${ }^{1}$, Pallab Kanti Podder ${ }^{2}$ \\ ${ }^{1}$ Department of Information and Communication Technology, Islamic University, \\ Kushtia-7003, Bangladesh \\ ${ }^{2}$ Department of Information and Communication Engineering, Pabna University of \\ Science and Technology, Pabna, Bangladesh
}

\begin{abstract}
The paper deals with the study based on the comparative analysis of radio propagation models for mobile cellular wireless communication of global system for mobile at frequencies $0.9 \mathrm{GHz}$ and $1.8 \mathrm{GHz}$, respectively. The path loss propagation models are vital tool for planning the wireless network as well as designed to predict path loss in a meticulous environment. Various propagation models: Free-space model, CCIR (ITU-R) model, Hata model, Ericson model, and Stanford University Interim (SUI) model have been studied and examined through analytically from the base station (BS) to mobile station (MS) and vice versa followed by respective simulation performance evaluation by using Matlab simulator. The observed data is collected at the operating frequency of $0.9 \mathrm{GHz}$ from various environments (high density region and low density region) using the spectrum analyzer and path loss comparison is shown for different model.
\end{abstract}

\section{KEYWORDS}

Mobile Cellular Communication, Path Loss, Free-Space model, Hata model, Stanford University Interim (SUI) model, Ericson model.

\section{INTRODUCTION}

For the past few years, mobile wireless communications have been developed quickly and making the mobile station (MS) an elemental part of the inhabitants in this universe. So, the market for mobile cellular wireless communications companies is rising gradually[1-3]. In wireless Communication system, path loss that occurs between transmitter and receiver and is a major factor in analyzing and designed for wireless communication system[4]. Mobile communication systm's prformanc is restricted by the radio channel and the signal propagation path from the transmitter to reciver and vice versa varies arbitrarily from simple line of sight (LOS) to obstruct harshly by building, foliage as well as any other obstacles that block the LOS path[5]. Multipath propagation occurs due to many rflection from the transmitter to receiver of the transmitted signal that cause variations in the receiver signal's phase and amplitude. There is no direct wave in both transmitter and receiver in the urban area. As a consequence, most cellular radio system is controlled by the multiple obstacles such as building, moving objects by diffraction, reflection and scattering[6]. For the design of a cellular wireless communication system, propagation models are very much significant and concentrate on predicting signal strength at the receiver. A general point to point mobile wireless communication system is 
illustrated in Fig.1. Besides, they depict a series of mathematical equations with algorithms are considered for the prediction of radio signal propagation in particular environment. In mobile cellular wireless communications, the propagation models are commonly used for evaluating feasibility studies of installing a base station (BS) to arbitraly mobile station (MS) where the system is suitable for operation and the location of transmitter and receiver. This study is focused on Free-space model, CCIR (ITU-R) model, Hata model, Ericson model, Stanford University Interim (SUI) model.

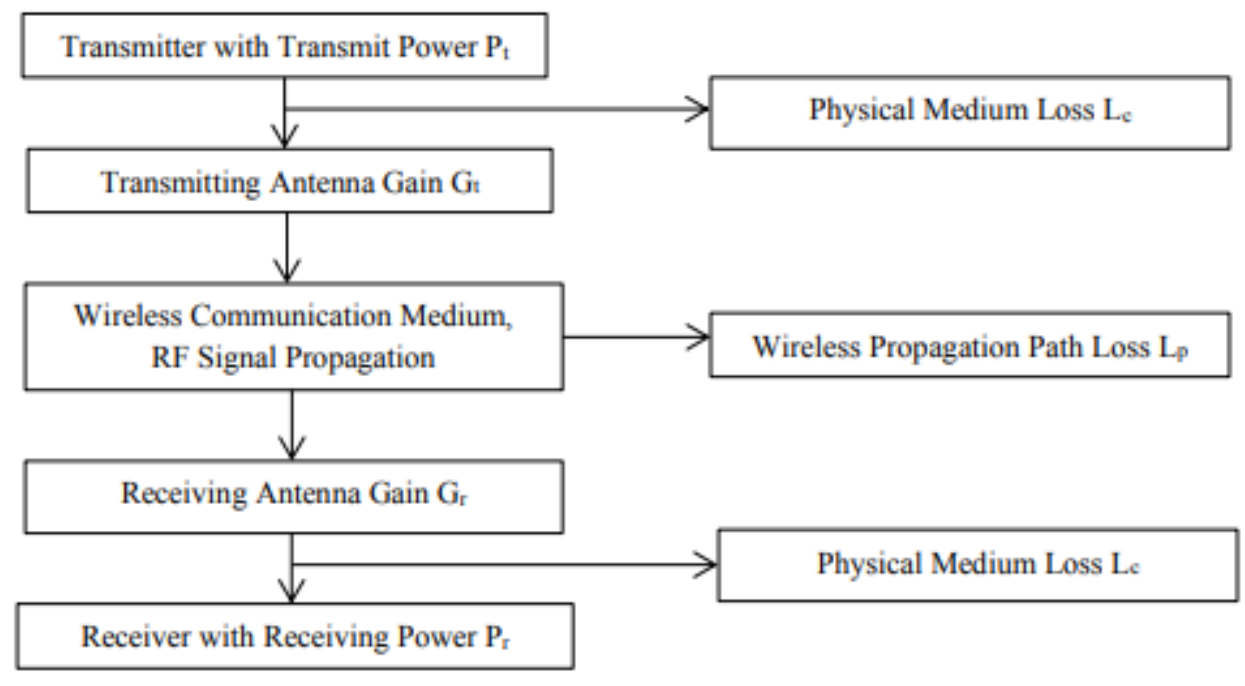

Fig.1. A general wireless communication model block diagram

\section{DifFerent Models}

\subsection{Model for Free-Space:}

The easiest path loss model is called free-space model which consider only frequency $f_{\text {in } М \text { Hz }}$ and distance $d i s_{k m}$ for taking into account. There are no obstacles and atmospheric effects in freespace propagation. Propagation path loss is obtained by using this model through the Eq. (1) [5].

$$
L_{F S P}=32.45+20 \log _{10}\left(d i s_{k m}\right)+20 \log _{10}\left(f_{i n M H z}\right)(1)
$$

\subsection{Model for CCIR (ITU-R):}

This model accepts terrain profile and it is caused path loss of free-space path loss[7]. The total path loss is obtained by using the Eq. (2).

$$
L_{I T U-R}=69.55+26.16 \log _{10}(f)-13.82 \log _{10}\left(h_{b s}\right)-a\left(h_{m s}\right)+\left[44.9-6.55 \log _{10}\left(h_{b s}\right)\right] \log _{10}\left(\operatorname{dis}_{k m}\right)-B(2)
$$

where, $a\left(h_{m s}\right)=\left[1.1 \log _{10}(f)-0.7\right] h_{m s}-\left[1.56 \log _{10}(f)-0.8\right]$ and $B=(\%$ area covered by building $)$.

\subsection{Model for Hata:}

This model is the experimental implementation of the information regarding path loss submitted by Okumara[5]. It is founded on the model of CCIR (ITU-R) and wide measurements of losses in both urban and suburban radio propagation. A basic formula established by this model for 
International Journal on Cybernetics \& Informatics (IJCI) Vol. 9, No.1/2/3, June 2020

calculating path loss in urban environment and revision equations for suburban as well as rural environments that is given by Eq. (3).

$$
L_{\text {hata }}(\text { urban })=69.55+26.16 \log _{10}(f)-13.82 \log _{10}\left(h_{\text {bse }}\right)-a\left(h_{\text {msant }}\right)+\left(44.9-6.55 \log _{10} h_{\text {bse }}\right) \log _{10}\left(d i s_{\text {km }}\right)(3)
$$

where, $L_{\text {hata }}=$ path loss for hata model in $\mathrm{dB}, f=$ carrier frequency in $\mathrm{MHz}, h_{b s e}=$ base station height (effective), $h_{m s a n t}=$ mobile station antenna height, $d i s_{k m}=$ distnace from transmitter to receiver in $\mathrm{km}$, and $a\left(h_{\text {msant }}\right)=$ correction factor for effective mobile station antenna height (function of the service area or city).

Correction factor of the effective mobile station antenna height for small or medium sized city is obtained by the Eq. (4).

$$
a\left(h_{\text {msant }}\right)=\left(1.1 \log _{10} f-0.7\right) h_{\text {msant }}-\left(1.56 \log _{10} f-0.8\right) d B(4)
$$

And for large city, this factor is obtained by the Eqs. (5) and (6).

$$
\begin{aligned}
& a\left(h_{\text {msant }}\right)=\left[8.29\left(\log _{10} 1.54 h_{\text {msant }}\right)^{2}-1.1\right] d B \text { with } f \leq 300 M H z(5) \\
& a\left(h_{\text {msant }}\right)=\left[3.2\left(\log _{10} 11.75 h_{\text {msant }}\right)^{2}-4.97\right] d B \text { with } f>300 M H z(6)
\end{aligned}
$$

For suburban and open (rural) areas, the path loss is obtained by the Eqs (7) and (8).

$$
\begin{gathered}
L_{\text {hata }}=L_{\text {hata }}(\text { urban })-2\left[\log _{10}(f / 28)\right]^{2}-5.4 d B(7) \\
L_{\text {hata }}=L_{\text {hata }}(\text { urban })-4.78\left(\log _{10} f\right)^{2}+18.33 \log _{10} f-40.98 d B(8)
\end{gathered}
$$

For carrier frequencies ranges $1500 \mathrm{MHz}$ to $2000 \mathrm{MHz}$, the path loss is obtained using hata model by the Eq. (9) [8].

$$
P L_{\text {hata }}(\text { urban })=46.3+33.9 \log _{10}(f)-13.82 \log _{10}\left(h_{\text {bse }}\right)-a\left(h_{\text {msant }}\right)+\left(44.9-6.55 \log _{10} h_{b s e}\right) \log _{10}\left(d i s_{k m}\right)+A(9)
$$

where $A(\mathrm{e})$ is $0 \mathrm{~dB}$ for medium sized cities and $3 \mathrm{~dB}$ for metropolitan cities. Such modified mathematical equations were successfully used at $1800 \mathrm{MHz}$ band for the design of cellular mobile radio networks. Modified Hata model however is appropriate for the design of macrocell $\left(d i s_{k m}>1 \mathrm{~km}\right)$.

\subsection{Model for Ericson:}

This model is established by the network designers who were used a software supplied by Ericson company[8]. It is also based on theupdated Okumara-Hata model to permit the room by vrying in parameters in consonance with the propagation environment. The path loss is obtained for this model by Eq. (10)[9].

$$
L_{\text {ericson }}=m_{0}+m_{1} \log _{10}\left(d i s_{\text {km }}\right)+m_{2} \log _{10}\left(h_{\text {beric }}\right)+m_{3} \log _{10}\left(h_{\text {beric }}\right) \cdot \log _{10}\left(d i s_{\text {km }}\right)-3.2\left[\log _{10}\left(11.75 h_{\text {reric }}\right)^{2}\right]+g_{\text {eric }}(f)(10
$$

where, $g_{\text {eric }}(f)=44.49 \log _{10}(f)-4.78\left[\log _{10}(f)\right]^{2}, f$ is the frequency in $\mathrm{MHz}, h_{\text {reric }}$ is the receiving antenna height in meters, $h_{\text {beric }}$ transmitting antenna height in meters, The values of these parameters $\left(m_{0} m_{1} m_{2} m_{3}\right)$ for different terrains are given in Table. $1[9,10]$. 
Table 1.Parameters values for Ericson model

\begin{tabular}{|c|c|c|c|c|}
\hline Terrain types & $m_{0}$ & $m_{1}$ & $m_{2}$ & $m_{3}$ \\
\hline Rural & 45.95 & 100.6 & 12 & 0.1 \\
\hline Suburban & 43.20 & 68.63 & 12 & 0.1 \\
\hline Urban & 36.20 & 30.20 & 12 & 0.1 \\
\hline
\end{tabular}

\subsection{Model for Stanford University Interim (SUI):}

A model is recommended by Stanford University called SUI model using the frequency band not higher than $11 \mathrm{GHz}$. The statistical analysis is obtained from this model for the $2.5 \mathrm{GHz}$ to 2.7 GHz frequency band of Multipoint Microwave Distribution System (MMDS). This model encompasses three types of terrain including $\mathrm{X}, \mathrm{Y}$, and Z. Hilly terrain with low to severe number of trees area is considered in Type $\mathrm{X}$ which has highest path loss. Type $\mathrm{Y}$ is defined by either mainly flat terrains with low to severe tree densities or hilly terrains with less number of tree densities. Type $\mathrm{Z}$ is for the smallest path loss and applicable to flat terrains with less number of tree densities. A simple path loss equation of this model with adjustment factors is demonstrated by the following Eq. (11) in [11, 12].

$$
L_{S U I}=A+10 \gamma \log _{10}\left[d i s / d i s_{0}\right]+f_{\text {correc }}+f_{h}+s \text { when } \text { dis }>\operatorname{dis}_{0}(11)
$$

Where, ' dis, is the separation between the antennas from transmitter to receiver, ' $d i s_{0}$ ' is $100 \mathrm{~m}$, ' $f_{h}$ ' is the antenna height measured in meters for adjusting the receiving antenna, ' $\gamma$ ' is an exponent of the path loss, ' $f_{\text {correc }}$ ' is the frequency adjustment level in $\mathrm{MHz}$, ' $s$ ' is the shadowing change in $\mathrm{dB}$ and the range of its values between $8.2 \mathrm{~dB}$ to $10.6 \mathrm{~dB}$ in the presence of trees and other make the propagation path mess [13]. Parameter $M$ is given by the Eq. (12).

$$
M=20 \log _{10}\left(4 \pi d i s_{0} / \lambda\right)
$$

The path loss exponent is calculated using by Eq. (13).

$$
\gamma_{\exp }=a-b h_{b s}+\left(c / h_{b s}\right)
$$

where $\lambda$ is the wavelength measured in meters, $h_{b s}$ is the antenna height for base station above the ground and the range of its values are $10 \mathrm{~m}$ to $80 \mathrm{~m}$. The three constants $\left(a_{\text {sui }}, b_{\text {sui }}, c_{\text {sui }}\right)$ rely on the various terrain that are specified in Table.2 [4].

Table.2 Different values for parameter of SUI model in different terrain

\begin{tabular}{|c|c|c|c|}
\hline Model's parameter & Terrain X & Terrain Y & Terrain Z \\
\hline$a_{\text {sui }}$ & 4.6 & 4.0 & 3.6 \\
\hline$b_{\text {sui }}\left(m^{-1}\right)$ & 0.0075 & 0.0065 & 0.005 \\
\hline$c_{\text {sui }}(\mathrm{m})$ & 12.6 & 17.1 & 20.0 \\
\hline
\end{tabular}

The adjustmenr factor is both for the height $f_{h}$ of the receiving antenna and operating frequency $f_{\text {correc }}$ for the SUI model are demonstrated in Eqs. (14) and (15)[13]. 
Terrain type $X$ and $Y$ :

$$
\begin{gathered}
f_{h}=-10.8 \log _{10}\left(h_{r} / 2000\right)(15) \\
f_{\text {correc }}=6 \log _{10}(f / 2000)(16)
\end{gathered}
$$

where $h_{r}$ is stand for the heightabove the ground level of thereceiving antennaand measuredin meters.

\section{Measured Data}

In this study, two stations (BS and MS) is considering to investigate the performance of different path loss models for mobile cellular wireless communications. The field strength of the transmitted signal from the BS to MS has taken by a handheld radio frequency (RF) field strength spectrum analyzer[14]. Path loss has been determined from estimated field strength values obtained.

\section{RESUlt AND Discussion}

The observed data have been collected in Nigerian's urban (high density region) and rural (low density zone) regions, making it more appropriate for urban (small and medium city size) 0.9 $\mathrm{GH}_{\mathrm{Z}}$ frequency band. Several selected propagation models are estimating using Mathlab simulation software MATLAB R2018a and compared with the experimental results that has taken in explicitly urban and rural environments. Transmitting antenna power is used $39 \mathrm{dBm}$, with BS antenna height $8 \mathrm{~m}$ as well as MS antenna height $1 \mathrm{~m}$ at frequencies $0.9 \mathrm{GHz}$ and $1.8 G H Z$ respectively. The path loss propagation simulation values of the four models as shown in Fig. 1 and Fig. 2 at frequencies $900 \mathrm{MHz}$ and $1800 \mathrm{MHz}$ bands, respectively.

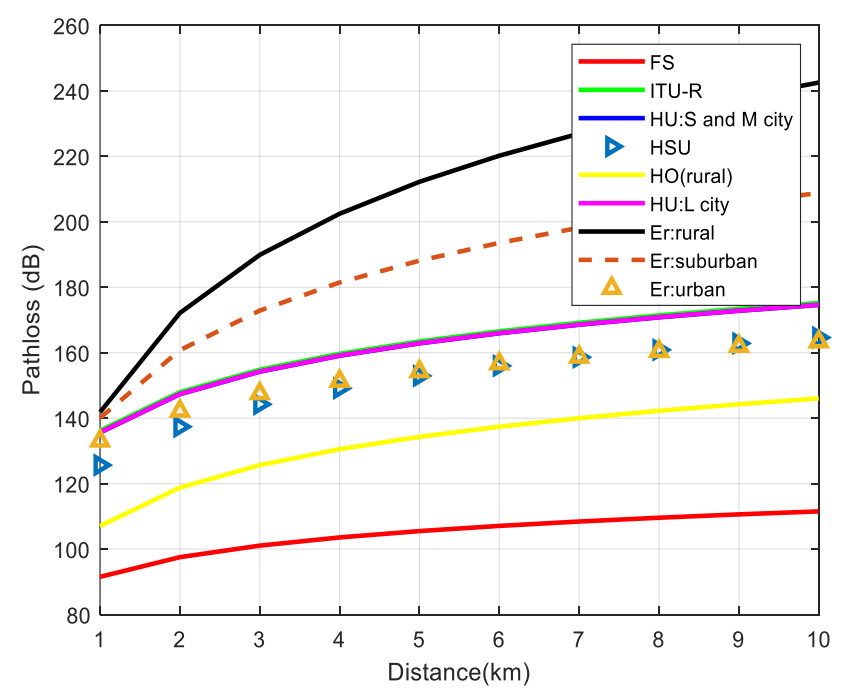

Fig. 1: Path loss comparison for four models with different environment (at $900 \mathrm{MHz}$ ) 
International Journal on Cybernetics \& Informatics (IJCI) Vol. 9, No.1/2/3, June 2020

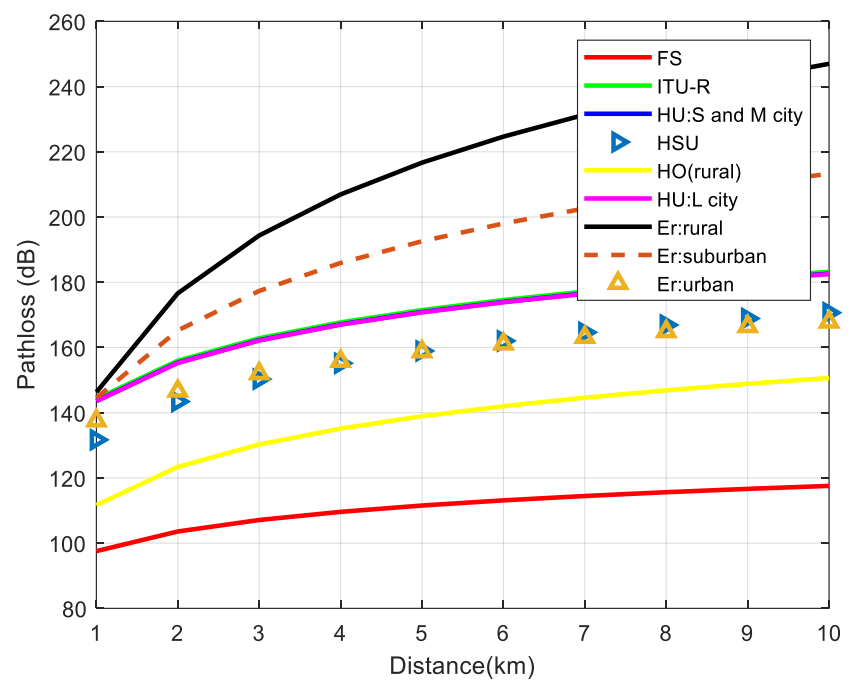

Fig. 2: Path loss comparison for four models with different environment (at $1800 \mathrm{MHz}$ )

It is notable that all path loss of the propagation model at frequency $1800 \mathrm{MHz}$ band is slightly higher than at frequency $900 \mathrm{MHz}$ band with the same values of other parameters in simulation.The propagation path loss in rural, suburban, and urban environments using the Ericson model shown in Fig.3. From the simulation result of this model, it observed that propagation path loss is less compared in urban environment than others two environments, namely: suburban and rural. So, this model is suitable for urban environment.

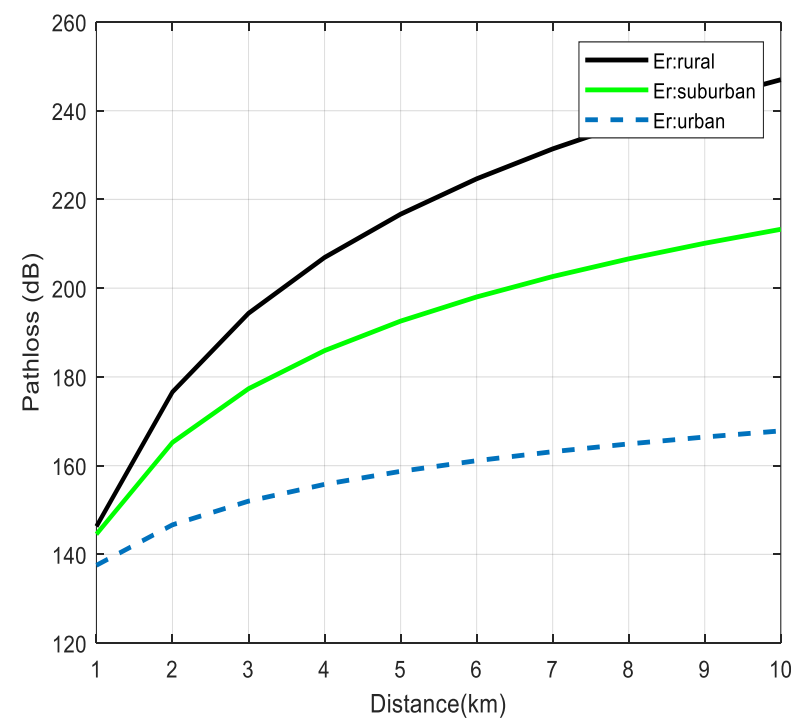

Fig. 3: Path loss comparison for Ericson model with different environment (at $1.8 \mathrm{GHz}$ )

Comparison of path losses for SUI model to various environments shown in Fig.4. Type ' $X$ ' and type ' $\mathrm{Y}$ ' terrain path loss is observing be almost identical with increasing the distance from transmitter to receiver. 
International Journal on Cybernetics \& Informatics (IJCI) Vol. 9, No.1/2/3, June 2020

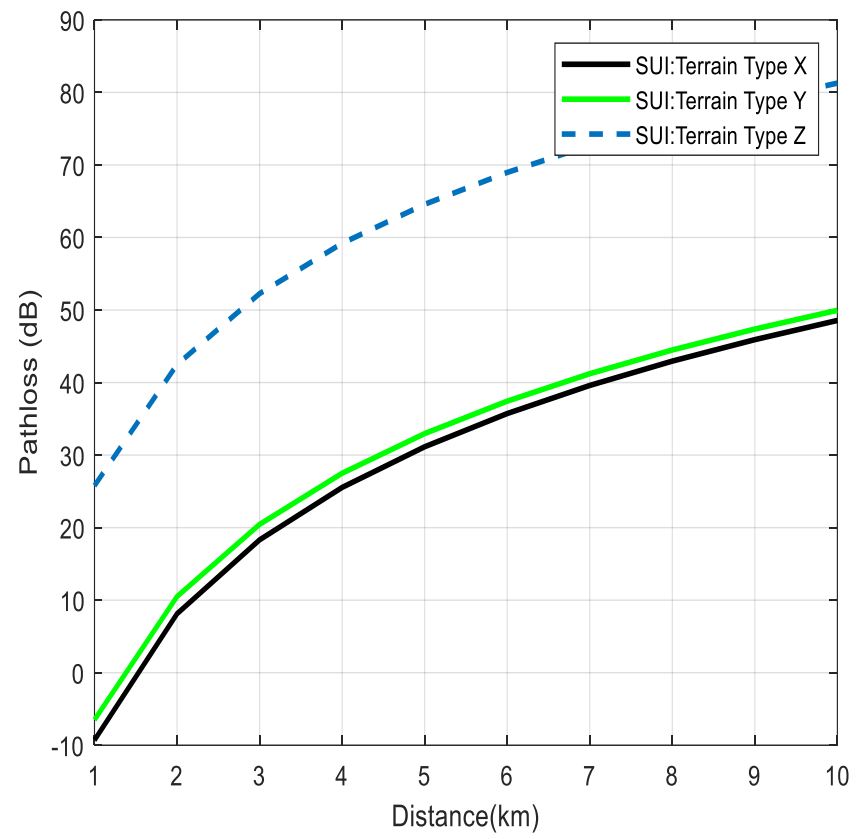

Fig. 4: Path loss comparison for SUI model with different environment (at $2.5 \mathrm{GHz}$ )

The measured path losses in different environments shown in Fig.5. From the observed path loss values, it is notable that $98 \mathrm{~dB}$ loss is same in $2.5 \mathrm{~km}$ distance at $0.9 \mathrm{GHz}$ frequency band for Hata model as well as Ericson model.

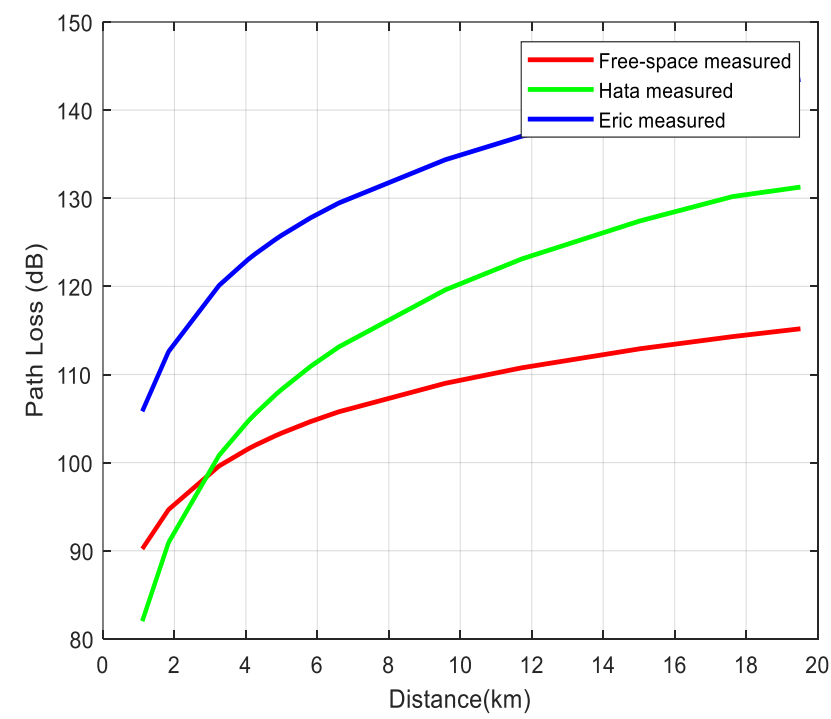

Fig. 5: Path loss comparison for Three models using measured data (at $0.9 \mathrm{GHz}$ )

Path loss comparison of calculated and measured values for two models, namely: free-space and Hata model shown in Fig.6. It is observed that calculated and measured values are almost same for free-space path loss model and slightly different for Hata model. 


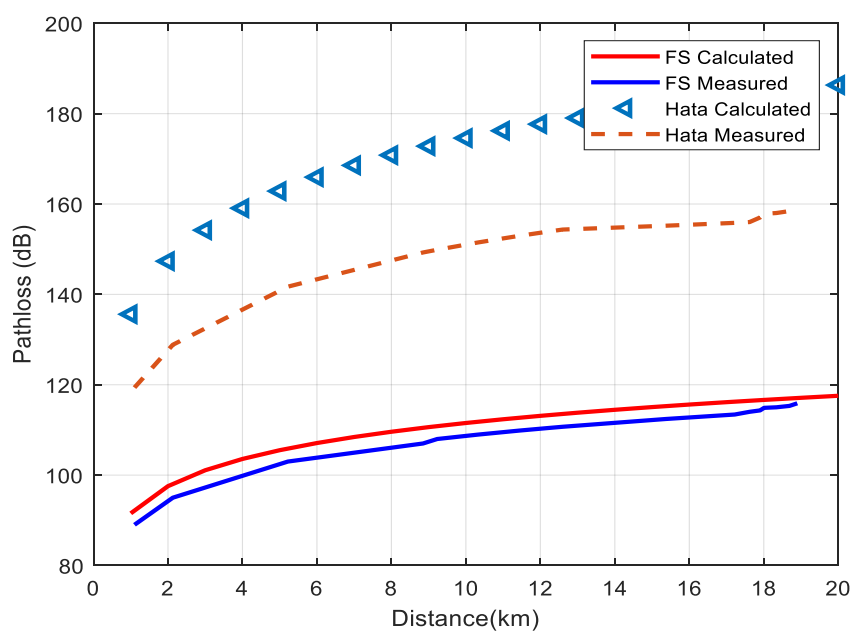

Fig. 6: Path loss comparison for two models (calculated vs measured)

\section{Conclusion}

The prime goal of this work is to study and analyze the proper model of path loss propagation in various environments in the mobile cellular wireless communication system. Compared with the $0.9 \mathrm{GHz}$ and $1.8 \mathrm{GHz}$ frequency bands in different environments, simulated and calculated measured path loss values of most suitable models are analyzed. However, to get better performance, the SUI model is analyzed at $2.5 \mathrm{GHz}$ to $2.7 \mathrm{GHz}$ frequencies in different types terrain. In this work, there are five models are analyzed: Free-space model, CCIR (ITU-R) model, Hata model, Ericson model, SUI model based on using same parameters except SUI model. From the simulation results, it is observed that Hata model is better predictive of rural as well as urban environments. Simulation and measured data comparison is not shown for all path loss model owing to not available measured data.

\section{REFERENCES}

[1] H. K. Hoomod, I. Al-Mejibli and A. I. Jabboory, "Analyzing Study of Path loss Propagation Models in Wireless Communications at 0.8 GHz", Journal of Physics: Conf. 2018.

[2] Z. Nadir, M. Bait-Suwailam, and M. Idrees, "Path loss measurements and Prediction using Statistical Models" in MATEC Web of Conference, 2016.

[3] H. K. Hoomod, I. Al-Mejibli and A. I. Jabboory,"Optimizing SOM for cell towers distribution" in New trends in Information \& Communications Technology Applications (NTICT), Annual Conference on Bagdad, pp. 138-143, 2017.

[4] Y. Zakaria, J. Hosek and J. Misurec, "Path Loss Measurements for Wireless Communication in Urban and Rural Environments", American Journal of Engineering and Applied Sciences, 2015, 8(1), pp. 94-99.

[5] R.K. Saha, "A report on path loss models used in mobile communications and a comparative analysis of these models for urban case using suitable parameters", Report on AT77.07: Cellular mobile systems, Asian Institute of Technology, Thailand, January 2010.

[6] T.S. Rappaport, "Wireless Communication", $1^{\text {st }}$ edition, Prentic Hall, Upper Saddle River, New Jersey, ISBN-10: 0-13-375536-3, pp: 641, 1996. 
[7] W. Debus, "RF path loss and transmission distance calculation", Technical Memoorendum, Axonn LLC, August 4, 2006.

[8] K.M. Ahmed, "Cellular Mobile Systems", Lecture notes: AT77.07, Asian Institute of Technology, Thailand, January 2010.

[9] J.S. Milanovic, R. Drlje and K. Bejuk, "Comparison of propagation model accuracy for WiMAX on $3.8 \mathrm{GHz}$ ", Proceedings of the $14^{\text {th }}$ IEEE International Conferrence on Electronic Circuits and Systems, Dec. 11-14, IEEE Xplore press, Morocco, pp. 111-114, 2007.

[10] I. L. Simic, I. Stanic and B. Zrnic, "Minimax LS algorithm for automatic propagation model tuning", Proceeding of the $9^{\text {th }}$ Telecommunications Forum, Nov. 20-22, Belgrade, Serbia, pp. 1-5, 2001.

[11] V. Erceg, L. J. Greenstein, S. Y. Tjandra, S. R. Parkoff and Gupta, et al., "An empirically based path loss model for wireless channels in suburban environments", IEEE J. Selected Areas Communications, 17, pp. 1205-1211, 1999.

[12] V. Erceg, K. Hari, M. S. Smith and D.S. Baum, "Channel models for fixed wireless applications", IEEE Tech. Report, 2001.

[13] V.S. Abhayawardhana, I.J. Wasell, D. Crosby, M.P. Sellars and M.G. Brown, "Comparison of empirical propagation path loss models for fixed wireless access systems", Proceedings of the 61th IEEE Vehicular Technolgy Conference, May-30 to June-1, IEEE Xplore press, Sweden, pp. 73-77, 2005.

[14] O.G. Igbinosa, U.K. Okpeki, "Performnce investigation of different pathloss models for a wireless communication system in Nigeria", Heliyon (5), 2019.

\section{Authors}

Md. Shariful Islam received the B.Sc. (Honors) and M.Sc. degrees from the Department of Electrical and Electronic Engineering (Erstwhile ofApplied Physics, Electronics and Communication Engineering) from Islamic University, Kushtia-7003, Bangladesh, in 1999(Examination held 2001) and 2000 (Examination held2003), respectively. He also received the $\mathrm{PhD}$ degree in 2016 from Islamic University, Kushtia-7003, Bangladesh. He joined as a lecturer in the Dept. of Information and Communication Technology, IU,Kushtia-7003, Bangladesh, in September 2004 and currently, he is working as Professor in the same department. His research interests are in the area of Integral Imaging 3D display technology. Image processing. Wireless Communications.

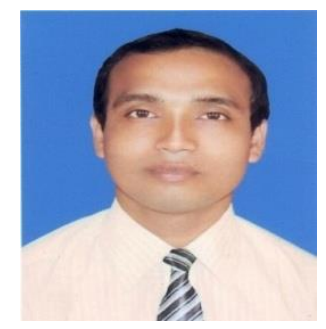

Md. AshekRaihan Mahmud received the B.Sc. (Engg.) from the Department of Electrical and Electronic Engineering from Rajshahi University of Engineering and Technology, Rajshahi, Bangladesh. He also received M.Sc. and M.Phil.from Australia (RMIT) as well as Islamic University, Kushtia-7003, Bangladesh. He joined as a lecturer in the Dept. of Information and Communication Technology, IU,Kushtia-7003, Bangladesh, in September 2004 and currently, he is working as an Associate Professor in the same department. His research interests are in the area of Mobile Ad-hoc Network, Telecommunication.

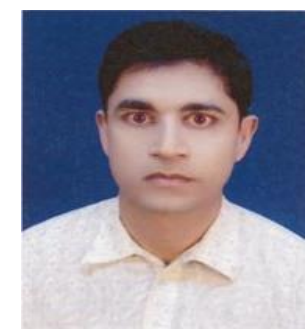


Md. Jashim Uddin received the B.Sc. (Honors) and M.Sc. from the Department of Information and Communication Technology, Islamic University, Kushtia7003, Bangladesh in 2005 and 2006 respectively. Presently, he is a PhD student on IoT. He joined as a lecturer in the Dapartment of Information and Communication Technology, Islamic University, Kushtia-7003, Bangladesh in 2010 and currently he is working as an Assistant Professor in the same department. His research interests are in the area of Internet of Things (IoT), Image and Speech Processing.

Dr. Pallab Podder (S'14 M'18) has completed his PhD from the CSU Machine Learning Research Unit of Charles Sturt University, Australia in December2017. Previously he completed Masters (M.Sc.) and B.Sc (Hons) degree and from the department Information \& Communication Engineering in 2010 and 2008 respectively. After his M.Sc. degree, he joined as a lecturer in the Computer Science \& Engineering Department of Bangladesh University, Dhaka, Bangladesh. Then he joined as a lecturer and promoted to Assistant Professor in the Department of Information \& Communication Engineering of Pabna Science \& Technology University, Pabna, which is one of the renowned universities in Bangladesh. After that, he has been serving as an Associate Professor and Chairman of the Information and Communicaton Engineering Department of the same University since 18 August 2018 and 28 September 2019 respectively. He has published more than 30 journal articles and International conference proceedings in the areas of communication, image processing, video compression, and video quality assessment. He is a member of the IEEE, Bangladesh Computer Council (BCC), and ACS (Australian Computer Society). 